\title{
Toward Time-Resolved Analysis of RNA Metabolism in Archaea Using 4-Thiouracil
}

\author{
Robert Knüppel¥, Corinna Kuttenberger ${ }^{\ddagger}$ and Sébastien Ferreira-Cerca* \\ Biochemistry III, Institute for Biochemistry, Genetics and Microbiology, University of Regensburg, Regensburg, Germany
}

Archaea are widespread organisms colonizing almost every habitat on Earth. However, the molecular biology of archaea still remains relatively uncharacterized. RNA metabolism is a central cellular process, which has been extensively analyzed in both bacteria and eukarya. In contrast, analysis of RNA metabolism dynamic in archaea has been limited to date. To facilitate analysis of the RNA metabolism dynamic at a system-wide scale in archaea, we have established non-radioactive pulse labeling of RNA, using the nucleotide analog 4-thiouracil (4TU) in two commonly used model archaea: the halophile Euryarchaeota Haloferax volcanii, and the thermo-acidophile Crenarchaeota Sulfolobus acidocaldarius. In this work, we show that 4TU pulse labeling can be efficiently performed in these two organisms in a dose- and time-dependent manner. In addition, our results suggest that uracil prototrophy had no critical impact on the overall 4TU incorporation in RNA molecules. Accordingly, our work suggests that 4TU incorporation can be widely performed in archaea, thereby expanding the molecular toolkit to analyze archaeal gene expression network dynamic in unprecedented detail.

\section{Keywords: archaea, RNA, 4-thiouracil, 4-thiouridine, 4TU, RNA-tagging, biotin}

\section{INTRODUCTION}

Determining the function of every single gene remains a challenging task for modern biology. The rise of -omics analyses has provided a great deal of information, which still needs to be integrated into meaningful cellular and molecular functions. In the post-genomic era, several functional analysis strategies can be applied to unravel the information stored at the DNA level. However, comprehensive functional analysis of individual genes contained in an individual genome still remains a daunting task (e.g., Bork et al., 1998; Niehrs and Pollet, 1999; Wu et al., 2002; Schlitt et al., 2003; Fraser and Marcotte, 2004). Moreover, complex phenotypic traits rely essentially on the establishment of highly cooperative and dynamic gene networks that can integrate the sum of functions of several individual gene products at a given time (e.g., Barabasi and Oltvai, 2004; Fraser and Marcotte, 2004). Whereas, classical functional genomic analysis can help to determine the function of a gene, such analysis often relies on prior knowledge and/or hypothetical projection (for example, predictions based on gene sequence similarities). Consequently, and despite full genome sequencing of numerous organisms, the function of many open reading frames remains poorly characterized.

System-wide profiling of gene expression and gene expression networks can aid in attributing functions to a gene, or groups of genes (e.g., Gardner et al., 2003; Ihmels et al., 2004; Blais and Dynlacht, 2005). Recently, the determination of the relative abundance of RNA by high-throughput technologies has revealed an unprecedented depth of information (Wang et al., 2009). Together, these key technologies provide fundamental information to predict and rationalize the molecular 
mechanisms that account for the formation of specific cellular phenotypes. However, the inherent lack of dynamic analysis in such steady-state assays does not grant full-depth understanding of the molecular basis enabling the formation of complex phenotypes (e.g., Friedel and Dolken, 2009).

To overcome such limitations, dynamic gene-expression network profiling has been applied to better characterize the gene expression networks accounting for the dynamic expression of complex traits in relation to environmental cues (e.g., Dölken et al., 2008; Zeiner et al., 2008; Friedel and Dolken, 2009; Miller et al., 2009; Tallafuss et al., 2014).

RNA-tagging approaches with the use of nucleotide/ nucleoside analogs, and in combination with next generation sequencing, is one of the recent technological developments that allows powerful analysis of RNA synthesis and degradation rates in a time- and condition-dependent manner. Nucleotide analogs, such as 4-thiouracil- (4TU) or 4-thiouridine- (4SU), -based RNA-tagging has been successfully applied in various cell types and tissues in combination with high-throughput methodologies (e.g., Favre et al., 1986; Dölken et al., 2008; Zeiner et al., 2008; Friedel and Dolken, 2009; Miller et al., 2009; Tallafuss et al., 2014). Together, these studies have provided essential information for further understanding of RNA metabolism and its dynamics (e.g., Swiatkowska et al., 2012; Burger et al., 2013; Barrass et al., 2015; Duffy et al., 2015; Hulscher et al., 2016). Moreover, the recent improvement of RNA-tagging chemistry enables a better enrichment of the tagged-RNA population providing additional perspectives to more precisely characterize gene-expression networks (Duffy et al., 2015). Furthermore, the promising usage of nucleotide analogs in combination with photo-crosslinking approaches allows the systematic analysis of RNA binding protein (RBP)-RNA interactions and to decipher the RBP repertoire (Hafner et al., 2010; Ascano et al., 2012; Castello et al., 2012). Whereas, nucleotide analog-based RNA-tagging has emerged as a key technology to systematically decipher fundamental aspects of RNA biology, surprisingly 4TU/4SU labeling, to our knowledge, has not been established in any archaea.

Since we are interested in deciphering key molecular principles of RNA metabolism in archaea, we sought to fill this methodological gap by first establishing and applying 4TU labeling in archaea. As a proof of principle we have employed 4TU labeling in two genetically-tractable representative archaeal organisms-Haloferax volcanii and Sulfolobus acidocaldariusthereby expanding the archaeal molecular biology toolkit.

\section{RESULTS}

\section{In vivo Incorporation of 4TU in Exemplary Archaea}

We initially speculated that efficient incorporation of 4TU into RNA molecules would largely depend on the following parameters: (i) efficiency of intra-cellular import of exogenous nucleobase, (ii) presence of enzyme(s) allowing conversion of the nucleobase into nucleotide tri-phosphate, (iii) and the existence of defined cultivation procedures where the nucleobase can be exogenously added.

On the basis of these criteria, we have selected widely used, easily cultivable model organisms representative of two major archaeal phyla: the Euryarchaeota Haloferax volcanii, and the Crenarchaeota Sulfolobus acidocaldarius. Both organisms can be cultivated aerobically in distinct media where the source of uracil can be exogenously added and controlled (Atomi et al., 2012). Moreover, in both organisms the pyrimidine biosynthesis pathway has been successfully mutated, whereby the respective pyrE genes encoding orotate phosphoribosyltransferase have been knocked out (Allers et al., 2004; Wagner et al., 2012). In this context, cells exclusively rely on an exogenous source of uracil to maintain growth.

Finally, conversion of the thiouracil into nucleotidetriphosphate (thio-UTP) is critically dependent on the uracil phophosribosyltransferase (EC: 2.4.2.9). In addition, in contrast to most multicellular eukaryotic organisms, with the exception of Toxoplasma gondii, only bacteria and unicellular eukaryotes have been shown to be able to convert thiouracil into thio-UMP, using their endogenous uracil phophosribosyltransferase (Cleary et al., 2005). As summarized in Figure 1, both organisms contain the annotated genes allowing the conversion of (thio-) uracil into (thio-) UTP (Kanehisa et al., 2016).

Since both organisms presented all the critical characteristics mentioned above to putatively allow efficient 4TU incorporation into RNA molecules, we next cultivated them in presence of 4TU over several growth generations to ensure sufficient intra-cellular accumulation of the nucleobase analog. Initially, we arbitrarily grew the cells in growth medium containing a 1:3 mixture of uracil and 4TU (75\% 4TU) respectively, (see Section "Materials and Methods"). Moreover, during the course of the experiments, the growth of the cells cultivated with or without 4TU was apparently indistinguishable at this working concentration (see below for detailed toxicity analysis). Nucleic-acids (total RNA) from cells grown in presence and absence of $4 \mathrm{TU}$ were extracted by hot-phenol/chloroform extraction (see Section "Materials and Methods"). The 4TU-labeled RNAs were then biotinylated (with HPDP-biotin or MTSEA-XX-biotin). Biotinylated-RNAs were immobilized on a nylon membrane prior to detection with the help of streptavidin conjugated to an infra-red dye and visualized on a LI-COR Odyssey imaging device (see Figure 1C for work flow and Section "Materials and Methods" for the detailed protocol).

As shown in Figure 1D, 4TU enrichment was observed in both organisms. Moreover, in agreement with a recent report (Duffy et al., 2015), the detection sensitivity was strongly enhanced by the use of MTSEA-biotin-XX (Supplementary Figure S1).

\section{Incorporation of 4TU into RNA Polymers}

Accumulation of intracellular $4 \mathrm{TU}$ is per se not an indication for proper incorporation of the nucleotide analog into functional RNA polymers. Therefore, to demonstrate incorporation of 4TU into RNA polymers, we monitored the presence of 4TU in stable abundant RNA (i.e., rRNA and tRNA). To this end, biotinylated total RNAs were separated by denaturing-agarose gel electrophoresis. As shown in Figure 2, the major stable 
A

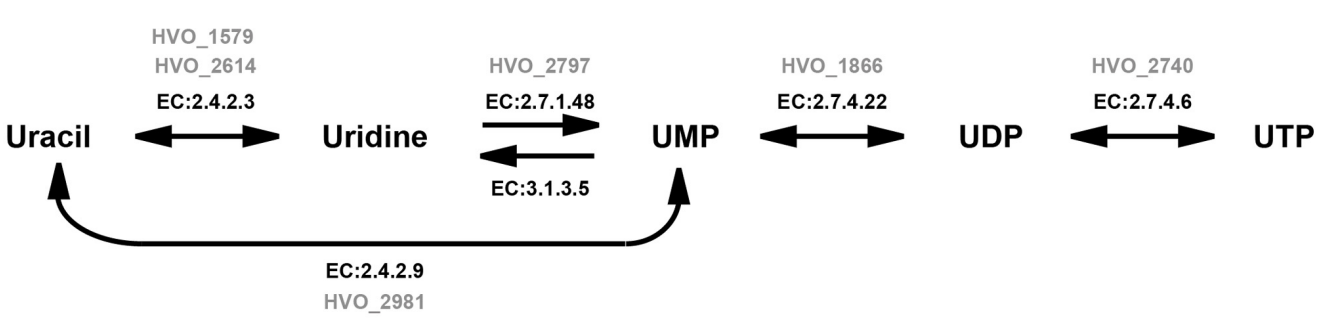

B

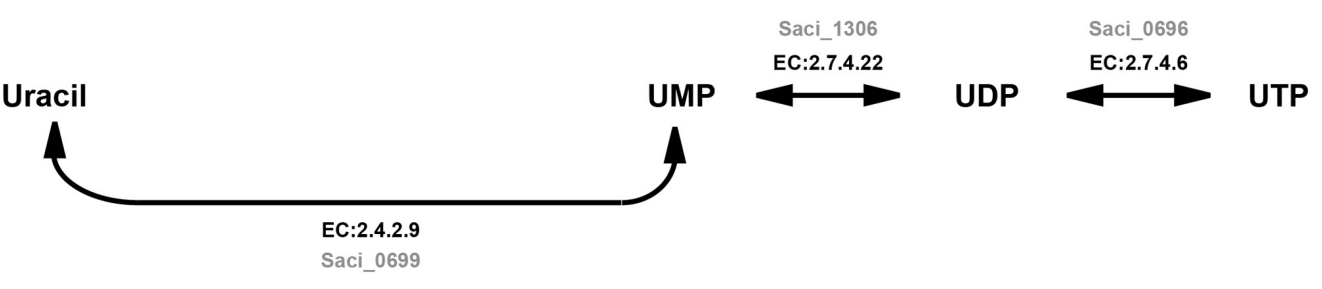

C

(1)

(2)

(3)

(4)

(5)

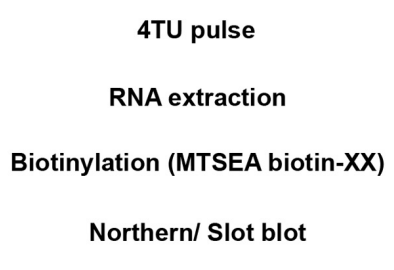

Detection (IR-Dye Streptavidin)

D

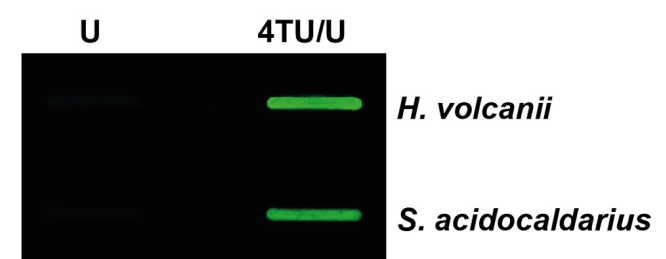

FIGURE 1 | In vivo incorporation of 4TU in model archaea. (A) Synthesis pathway of UTP from uracil in Haloferax volcanii is depicted according to KEGG pyrimidine synthesis pathway (KEGG entry: hvo00240) (Kanehisa et al., 2016). Enzyme classification number (E.C) and their corresponding open reading frame in H. volcanii (in gray) encoding the enzyme activity are indicated. (B) Synthesis pathway of UTP from uracil in Sulfolobus acidocaldarius is depicted according to KEGG pyrimidine synthesis pathway (KEGG entry: sai00240) (Kanehisa et al., 2016). Enzyme classification number (E.C) and their corresponding open reading frame in S. acidocaldarius (in gray) encoding the enzyme activity are indicated. (C) 4-thiouracil (4TU) labeling and detection work flow. (D) Analysis of 4TU incorporation in H. volcanii and S. acidocaldarius. H. volcanii (H26) and S. acidocaldarius (MW001) cells were grown for several generations either in medium containing a mixture of 4-thiouracil and uracil (4TU/U - 3:1) or in medium solely containing uracil (U) as described in the Section "Materials and Methods". Biotinylated uracil was detected by infra-red fluorescence.

RNAs are readily labeled by $4 \mathrm{TU}$. Collectively, these experiments demonstrate the feasibility to perform $4 \mathrm{TU}$ incorporation into RNA molecules in archaea.

\section{TU is Toxic at Higher Concentrations}

A previous report in mammalian cells demonstrated the potential toxic effect of $4 \mathrm{TU}$ incorporation on cellular growth (Burger et al., 2013). To evaluate this possibility, we grew $H$. volcanii and $S$. acidocaldarius cells in conditions where the final concentration of uracil-(analog) source (uracil/4TU) was constant, whereas the ratio of uracil/4TU was varied. $H$. volcanii growth curves are depicted in Figure 3A, and were generated in 96-well plate format [as described in Jantzer et al. (2011) and Section "Materials and Methods"]. In contrast, S. acidocaldarius growth analyses were performed manually and are depicted in Figure 3B. These growth analyses demonstrate little to no effect on growth behavior to up to $80 \%$ of $4 \mathrm{TU}$ as total source of uracil for both organisms. However, higher concentrations of 4TU significantly inhibited growth of both $H$. volcanii and $S$. acidocaldarius. This suggests that higher concentrations of 4TU are toxic. Therefore, for our subsequent analysis, we used concentrations of $4 \mathrm{TU}$ equal to or below $75 \%$ of the total uracil source.

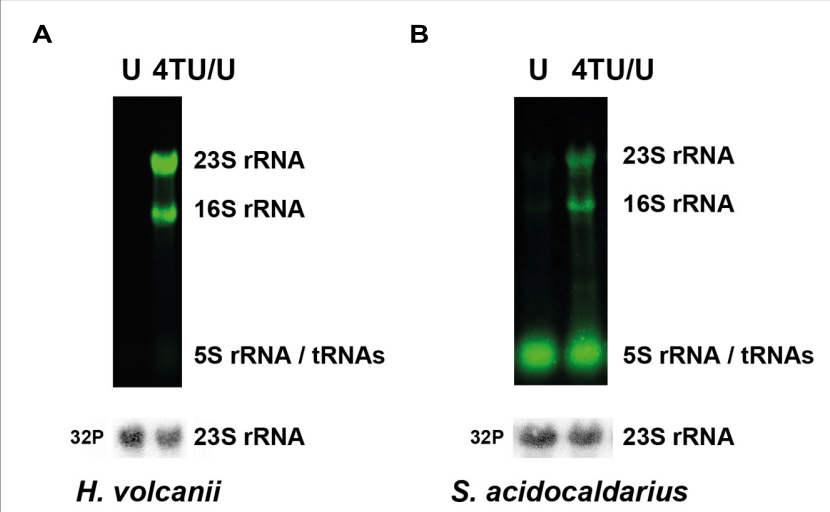

FIGURE 2 | In vivo incorporation of 4TU in RNA polymers. Steady-state incorporation of 4TU in abundant RNA molecules (rRNAs and tRNAs) in (A) H. volcanii and (B) S. acidocaldarius is depicted. Cells were grown over-night in culture medium either solely containing uracil $(U)$ or containing a defined mixture of 4-thiouracil and uracil (4TU/U - 3:1). Abundant 4TU-containing RNA species were detected by infra-red fluorescence (upper panel - green). Bulk steady-state 23S rRNA (used as loading control) was detected using ${ }^{32} \mathrm{P}$ radiolabeled antisense oligo probes (lower panel - gray) as described in the Section "Materials and Methods". 
A

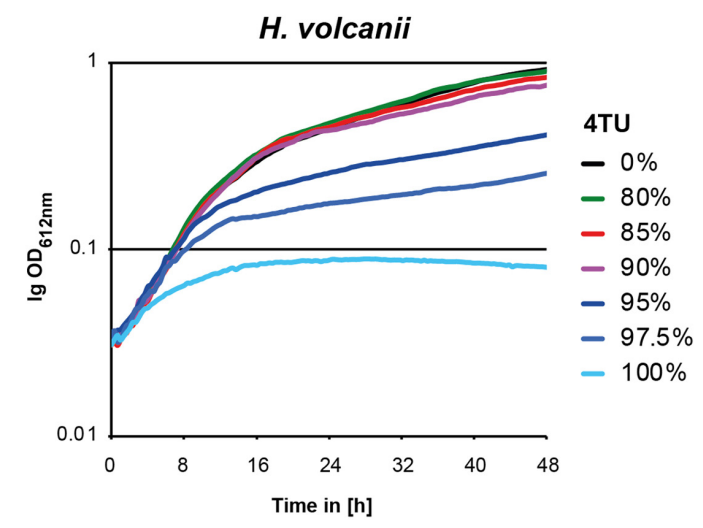

B

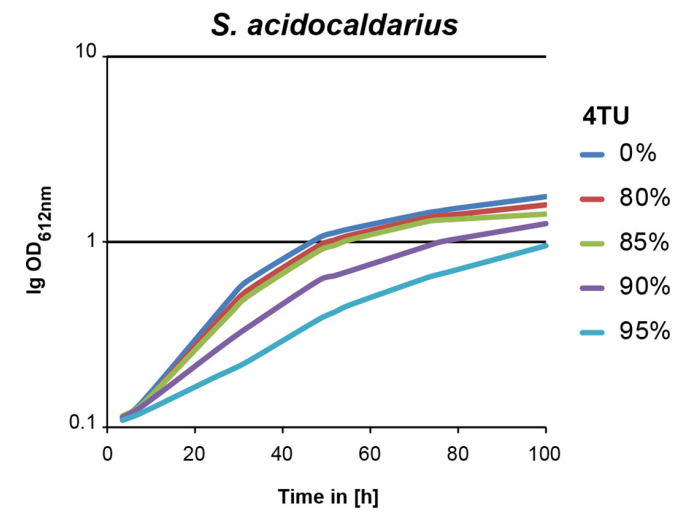

FIGURE 3 | In vivo toxicity of 4TU in model archaea. Growth analysis of (A) H. volcanii and (B) S. acidocaldarius grown in presence of different ratios of 4TU to uracil are depicted. Logarithmically growing cells were inoculated in culture medium containing the indicated amounts (in \%) of 4TU/uracil. Growth was further monitored by measuring optical density at regular time intervals over the depicted time. Representative results are provided.

\section{Time-Dependent 4TU Labeling of Major Cellular RNA}

One critical advantage of $4 \mathrm{TU}$ labeling is the possibility to perform pulse and pulse-chase labeling, thereby allowing time-dependent analysis of RNA synthesis/decay at a given condition. To evaluate this opportunity, we performed pulse and pulse-chase labeling in $H$. volcanii and $S$. acidocaldarius (Figures 4 and 5, respectively). Interestingly, in $H$. volcanii, 4TU incorporation in the large rRNA (16S and 23S) was detectable at a relatively short-time point (30 $\mathrm{min} ; 10-15 \%$ of a typical cell cycle in these growth conditions), which strongly accumulated over time (Figure 4). Likewise, pulse and pulse-chase experiments were performed in S. acidocaldarius (Figure 5). However, we noticed the presence of a stable population of shorter RNAs, presumably containing thio-modified nucleoside residues in this organism (see Figure 5 and Section "Discussion"). Finally, pulse-chase experiments demonstrated a relative decrease of the labeled rRNA over time (Figures 4 and 5). Altogether, these results highlight the feasibility of performing $4 \mathrm{TU}$-based timedependent analysis of RNA metabolism in archaea.

\section{Relative 4TU-Labeling Efficiency}

In our labeling experiments, we noticed that the apparent labeling efficiency in S. acidocaldarius was reduced as compared to that in $H$. volcanii. However, since the cells had different doubling times ( $\sim 3 \mathrm{~h}$ for $H$. volcanii and $\sim 9 \mathrm{~h}$ for $S$. acidocaldarius) in these growth conditions, it was difficult to perform an accurate comparison of the $4 \mathrm{TU}$ labeling efficiencies in the conditions described above. Therefore, to perform a fair comparison of the relative 4TU labeling efficiencies in these organisms we sought to minimize the growth rate-dependent effects on the overall incorporation efficiency. To this end, we labeled cells during one and two full generation times. In addition, equal amounts of total RNA were analyzed. As shown in Figure 6, the total fraction of labeled rRNA present in S. acidocaldarius is significantly reduced compared to that in $H$. volcanii. This result indicates, that despite successful labeling of both organisms, the overall labeling efficiencies may vary between different archaea.

\section{Optimal 4TU Concentrations}

Next, we sought to further optimize the 4TU labeling conditions. For this purpose, we analyzed the influence of various $4 \mathrm{TU}$ concentrations on the detection of synthesized tagged-RNA in $H$. volcanii. To this end, time-dependent pulse labeling with various amounts of $4 \mathrm{TU}(25,50$, and $75 \%$ of total uracil source) was performed (Figure 7). This analysis shows that within the non-toxic range of $4 \mathrm{TU}$ concentrations tested, the use of $75 \%$ of $4 \mathrm{TU}$ as total uracil allows robust labeling of RNA.

\section{Influence of Pyrimidine de Novo Synthesis on Overall 4TU Incorporation Efficiency}

Cells used in this study have been engineered by genomic deletion of the pyrE gene essential for de novo synthesis of pyrimidine. However, for many other archaea uracil auxotroph strains are not always available. The presence of a de novo synthesis pathway could lead to a lower usage efficiency of the extra-cellular uracil/4TU. Therefore, we evaluated incorporation of $4 \mathrm{TU}$ in a situation where the pyrE gene, and thus the pyrimidine de novo synthesis pathway, is restored (see Figure 8). We derived an H26 strain where the pyrE2 gene has been genomically integrated at a heterologous locus (see Section "Materials and Methods" and Supplementary Figure S2). The resulting uracil prototroph cells were grown in medium lacking uracil prior to addition of $4 \mathrm{TU} / \mathrm{U}$ (3:1) and aliquots were taken at the indicated time points. As shown in Figure 8, labeling of uracil prototroph strain efficiency was similar to that obtained in the isogenic uracil auxotroph strain. Therefore, we conclude that $4 \mathrm{TU}$ labeling can be potentially widely achieved in most archaea that can be grown under defined laboratory conditions.

\section{Analysis of the Relative Transcriptional Activity of a Regulated mRNA, Using 4TU}

4TU labeling has been widely applied for the analysis of shortlived and/or less abundant transcripts such as mRNAs. In this case, the 4TU-labeled RNA needs to be enriched by affinity purification before analysis. To ensure that $4 \mathrm{TU}$ pulse labeling 
A

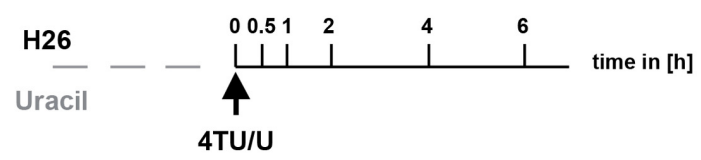

B
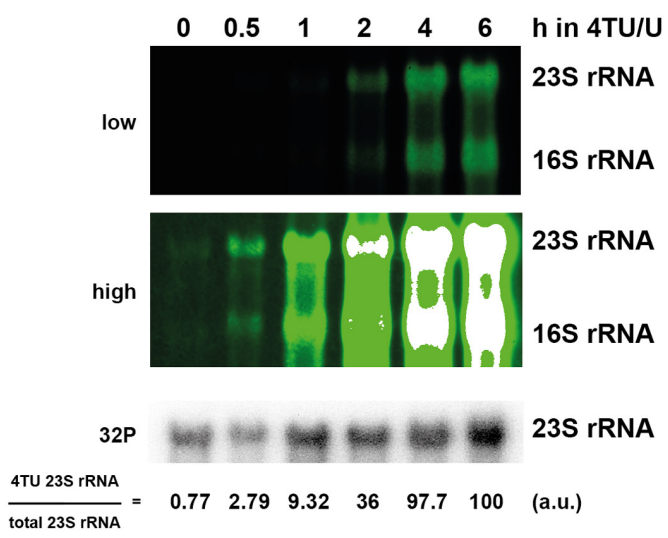

C

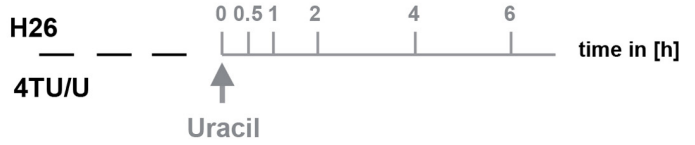

D
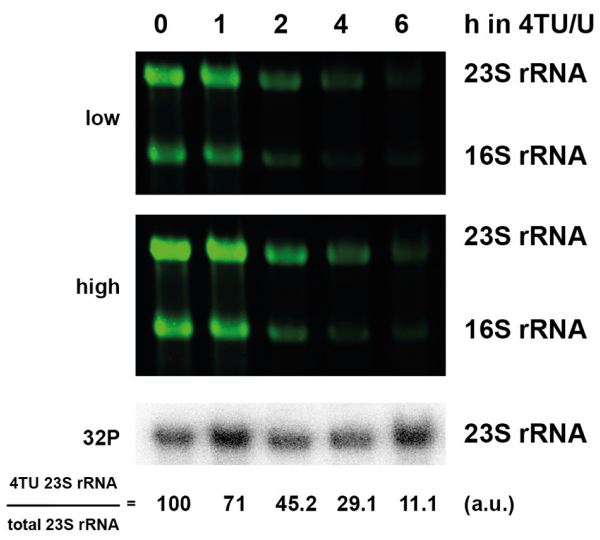

FIGURE 4 | Time-dependent incorporation of 4TU in $\boldsymbol{H}$. volcanii RNA molecules. 4TU pulse and pulse-chase experiments in $H$. volcanii are depicted in panel (A-D), respectively. (A) 4TU pulse-labeling experimental work flow. Logarithmically growing cells were transferred in pre-warmed culture medium containing 75\% of $4 \mathrm{TU}$ as uracil source $(t=0)$. Aliquots were collected at the indicated time points after addition of 4TU. (B) Time-dependent incorporation of 4TU in 16S and $23 \mathrm{~S}$ rRNA. Bio-tagged-RNAs and bulk 23S rRNA (upper panels - in green and lowest panel - in gray, respectively) were visualized and quantified as described in the Section "Materials and Methods". Two different acquisition sensitivities of the fluorescent signals are shown (low and high). Note that the white signals indicate fluorescence signal acquisition saturation. (C) 4TU pulse-chase labeling experimental work flow. H. volcanii cells were kept logarithmically growing in culture medium containing $75 \%$ of $4 \mathrm{TU}$ as total uracil source for $18 \mathrm{~h}$ to ensure labeling of most of the stable rRNA molecules $(t=0)$. Cells were then transferred to medium lacking 4TU and harvested at the indicated time points. (D) Time-dependent decrease of 4TU incorporation in 16S and 235 rRNA. RNA was analyzed as described in (B). (a.u.) arbitrary units.

can be applied for the analysis of archaeal mRNA we analyzed the transcriptional response of the regulated mRNA encoding tryptophanase (Large et al., 2007). This previous study has shown that upon addition of L-Tryptophan transcription of the tryptophanase gene is up-regulated (Large et al., 2007). Using this regulated mRNA as a model system, we performed 4TU pulse labeling over the course of addition of L-Tryptophan (Figure 9A). As shown in Figure 9 the relative amount of the tryptophanase mRNA in the 4TU-enriched RNA fraction (Figure 9C - right panel) mirrors the one observed in the total RNA fraction (Input) (Figure 9B). Together, these results show that the transcriptional state of 4TU-labeled mRNA can also be analyzed in archaea.

\section{DISCUSSION}

In this work, we provide proof of principle for the application of 4TU-based RNA-tagging in archaea. Given the known advantages of the methodology to analyze RNA dynamics and, RNA-protein interactions (e.g., Hafner et al., 2010; Ascano et al., 2012; Castello et al., 2012; Swiatkowska et al., 2012; Tallafuss et al., 2014; Duffy et al., 2015; Hulscher et al., 2016), we are confident that our proof of principle analysis broadens the archaeal molecular biology toolkit and will stimulate deeper analysis of RNA dynamics and gene expression networks in archaea.
We provide evidence that time-dependent 4TU based RNAtagging can be widely applied in different archaeal organisms independently of their uracil auxotrophy or prototrophy. However, one of the most critical parameters allowing efficient 4TU labeling appears to be the ability to grow the corresponding archaeal organisms in controlled drop-out medium lacking uracil. Moreover, the intrinsic 4TU uptake and/ or stability of the nucleotide analog in a particular growth conditions might influence the overall labeling efficiencies.

Whereas high amounts of $4 \mathrm{TU}$ provided in the culture medium are apparently well-tolerated in $H$. volcanii and $S$. acidocaldarius, it should be noted that, owing to the inherent toxicity of $4 \mathrm{TU}$, the ratio of $4 \mathrm{TU}$ to uracil chosen is critical. A previous study, has suggested that higher amounts of 4-thiouridine provided to human cell culture models have toxic effects, whereby early steps of ribosome synthesis are affected (Burger et al., 2013). Moreover, deleterious cross-talk effects of 4TU on naturally occurring uridine modifications (e.g., thiouridine derivatives, pseudouridine) have not been properly evaluated to date. Therefore, toxicity behavior for the respective organisms should be first determined empirically.

Another possible limitation of 4TU-based RNA tagging is the presence of free sulfhydryl groups, mostly found in the form of thiouridine modifications in tRNAs (2- and 4-thiouridine and their derivatives) (Tomikawa et al., 2013; Machnicka et al., 2014). 
A

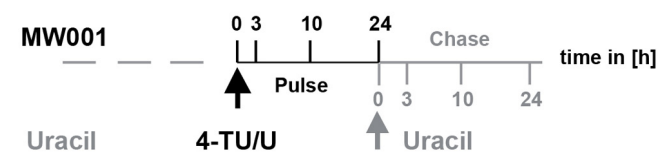

B

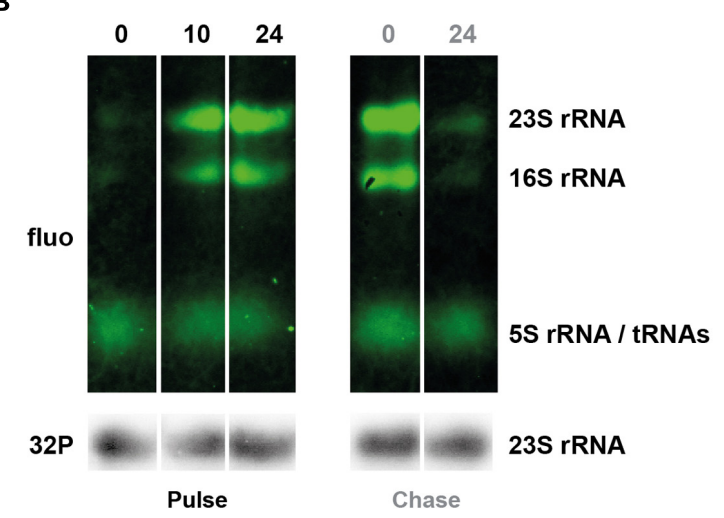

C

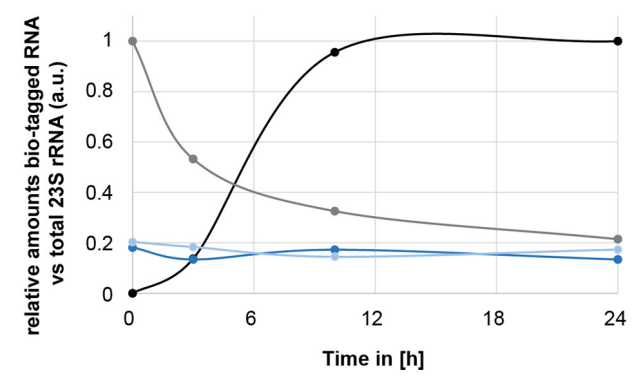

$\rightarrow$ Pulse 4TU/U 23S rRNA (fluo) / total 23S rRNA

$\rightarrow$ Chase 23S rRNA (fluo) / total 23S rRNA

$\rightarrow$ Pulse 4TU/U 5S rRNA tRNAs / total 23S rRNA

$\rightarrow-$ Chase 5 S rRNA tRNAs / total 23S rRNA

FIGURE 5 | Time-dependent incorporation of 4TU in S. acidocaldarius RNA molecules. (A) 4TU pulse- and chase-labeling experimental work flow. Logarithmically growing cells were first labeled with 4TU for $24 \mathrm{~h}$ (Pulse) and chased for another $24 \mathrm{~h}$ (Chase). Aliquots were collected at the indicated time points. (B) Time-dependent incorporation of $4 T \mathrm{TU}$ in stabile RNA. Bio-tagged RNAs (upper panels - in green) and total 23S rRNA (lower panel - in gray) were visualized and quantified as described in the Section "Materials and Methods". Representative time-points are provided. (C) Relative quantification of the major bio-tagged RNA in S. acidocaldarius. Relative amounts of bio-tagged RNA species after 4TU pulse-labeling and chase were quantified overtime in relation to total 23S rRNA (quantified from data depicted in (B) and data not shown).

In this case, the presence of endogenous sulfur-modified nucleosides will react with the modified biotin moiety and can potentially interfere with downstream experiments. Therefore, background reactivity should be carefully evaluated first. However, the complement and repartition of tRNA modifications in archaea is not yet fully characterized. Interestingly, whereas 4-thiouridine modification was not detected in $H$. volcanii tRNAs (Phillips and de Crécy-Lagard, 2011), 2-thiouridine modifications occurring at the wobble position of tRNA for
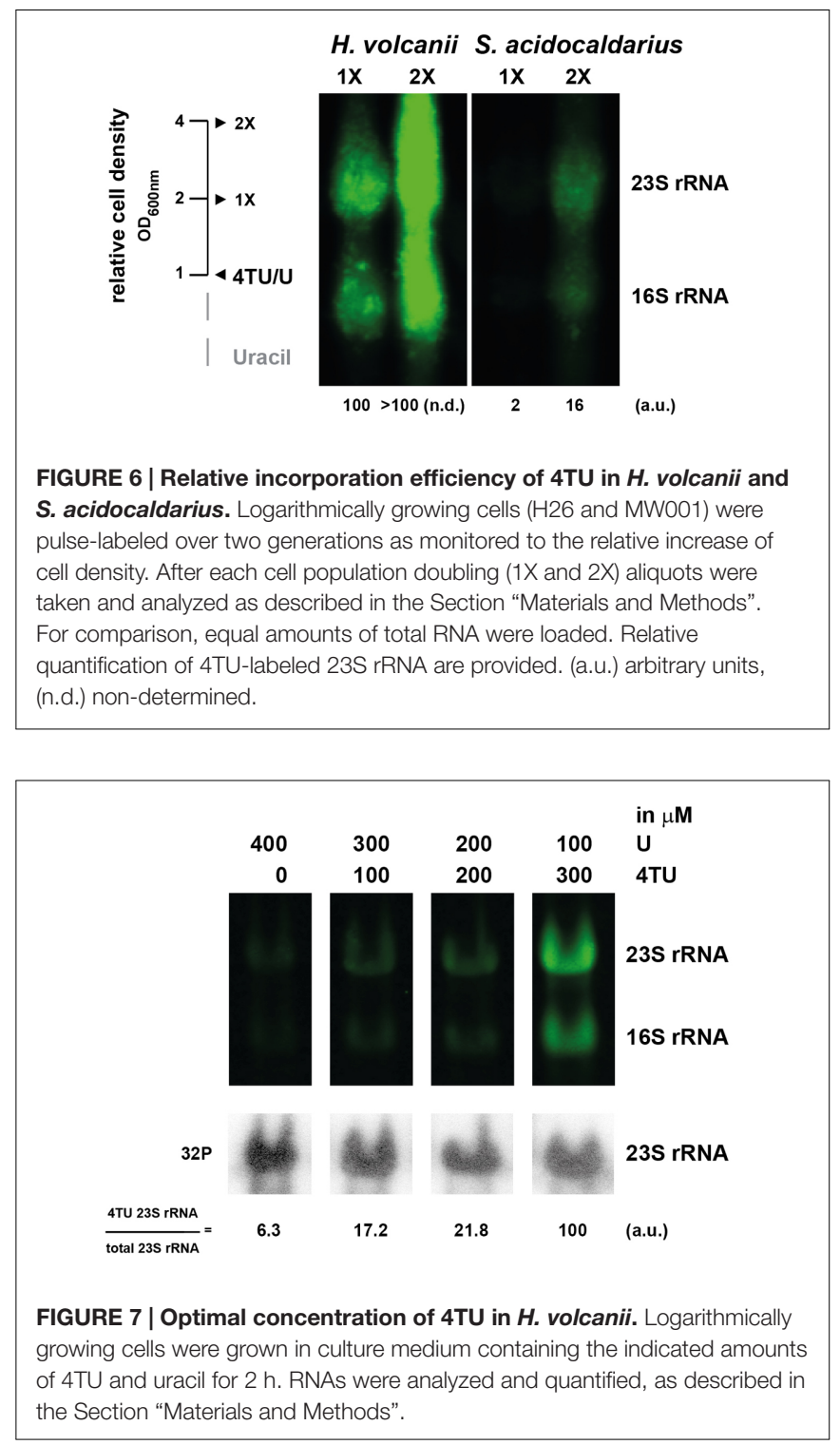

lysine (tRNA ${ }^{\text {Lys }}$ UUU $)$, glutamate ( $\mathrm{tRNA}^{\mathrm{Glu}} \mathrm{UUC}$ ), and glutamine (tRNA ${ }^{\text {Gln }}{ }_{U U G}$ ) have been only formally established for tRNA $^{\text {Glu }}{ }_{\text {UUC }}$ and tRNA ${ }^{\text {Lys }}$ UUU in $H$. volcanii (Rogers et al., 1995; Miranda et al., 2011; Chavarria et al., 2014; Shigi, 2014). In agreement, we have not observed significant labeling of tRNA in absence of $4 \mathrm{TU}$ in $H$. volcanii (see Figure 2A). In contrast, a substantial population of sulfur-modified (t)RNA was detected in the absence of 4TU labeling in S. acidocaldarius (Figures 2B and 5). As described above, 2-thiouridine modification is known to be restricted to a small population of tRNAs, whereas most bacterial tRNAs contain 4-thiouridine (Rogers et al., 1995). Therefore, the relative high amounts of thiolated tRNA could reflect a high steady-state level of endogenous 4-thiouridinemodified tRNA in $S$. acidocaldarius. The biosynthesis of 4-thiouridine is still not completely resolved in archaea. Recently, a conserved CXXC motif located in the PP-loop domain of Methanococcus maripaludis ThiI (MMP1354) has been shown 
A

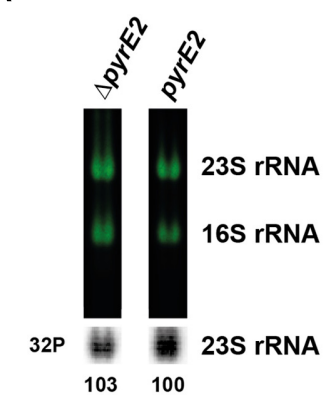

B

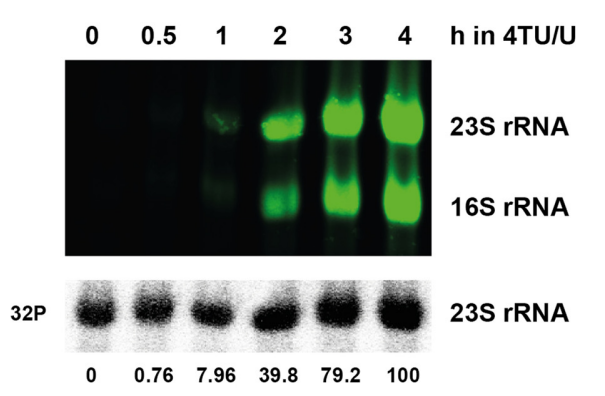

C

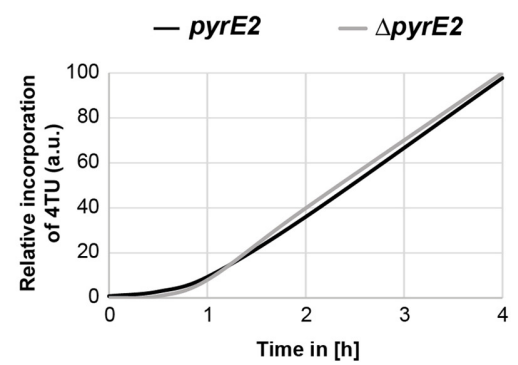

FIGURE 8 | Influence of pyrimidine de novo synthesis on overall 4TU incorporation. (A) 4TU incorporation in uracil auxotroph and prototroph strains. Uracil auxotroph and prototroph (pyrE2::HVO_0569) strains growing in $\mathrm{Hv}$-Ca+ medium supplemented or lacking uracil, respectively, were transferred for $8 \mathrm{~h}$ in pre-warmed $\mathrm{Hv}_{\mathrm{C}} \mathrm{Ca}^{+}$medium containing 75\% 4TU as uracil source. RNA were analyzed and quantified as described in the Section "Materials and Methods". Results of relative quantification (4TU-labeled 23S rRNA/total 23S rRNA) are provided (B) Time-dependent incorporation of 4TU in 16S and $23 \mathrm{~S}$ rRNA in uracil prototroph strain. Same as in (A), except that cells were grown in pre-warmed $\mathrm{Hv}$-Ca+ medium containing $75 \% 4 T \mathrm{~T}$ as uracil source and harvested at the indicated time-points. (C) Relative 4TU incorporation efficiency in auxotroph and prototroph cells. Results of relative quantification of $4 T U$ incorporation in $23 S$ rRNA from uracil auxotroph ( $\triangle$ pyrE2) (taken from Figure 4B) and from uracil prototroph (pyrE2::HVO_0569) (Figure 8B) strains are depicted.

to be essential for both in vitro and in vivo 4-thiouridine formation (Liu et al., 2012). Surprisingly, Halobacteriales and Sulfolobales are missing this critical CXXC motif (Liu et al., 2012; Tomikawa et al., 2013), suggesting that this group of organisms either lack 4-thiouridine modification (as demonstrated in $H$. volcanii) or use different or unknown mechanisms for the formation of 4-thiouridine. Another possibility is that $S$. acidocaldarius contains other type(s) of sulfur-containing nucleosides (e.g., 2-thioribothymidine) that contribute to its high-temperature adaptation (Edmonds et al., 1991; Shigi, 2014). Future studies will be needed to establish the nature and targets of the thio-modification observed in S. acidocaldarius.

Finally, biorthogonal amino acid pulse-labeling in combination with fluorescence in situ hybridization was developed to characterize cell-specific translational activity within complex environmental samples (Hatzenpichler et al., 2016, 2014). Whereas, we show that 4TU based RNAtagging seems to be amenable for most organisms grown under laboratory conditions, we speculate that 4TU labeling could be also used beyond its classical "laboratory usage" to characterize microbial consortia. In this context, using advantages of 4TU-tagged RNA sequencing analysis would provide complementary information about the organism-specific rate of rRNA synthesis, thereby shedding light on both the composition and the relative growth dynamic of the microbial consortia.

\section{CONCLUSION}

We described, proof of principle and workflow allowing the analysis of the RNA dynamic and gene network regulation in a time-dependent manner in archaea. The versatility and robustness of 4TU-based RNA-tagging can now be implemented in archaea and will further allow to elucidate the archaeal RNA metabolism.

\section{MATERIALS AND METHODS}

\section{Strains, Plasmids, and Growth Conditions}

Haloferax volcanii strains (H26 $\Delta$ pyrE2 - (Allers et al., 2004) and pyrE2::HVO_0569 pop-in, this work) were grown in enhanced casamino acids media $\left[\mathrm{Hv}-\mathrm{Ca}^{+}: \mathrm{Hv}\right.$-casamino acids medium supplemented with carbon source (Allers et al., 2004)] at $42^{\circ} \mathrm{C}$ under vigorous agitation. S. acidocaldarius [MW001 $\triangle p y r E-$ (Wagner et al., 2012)] cells were grown in standard Brock medium (Brock et al., 1972; Wagner et al., 2012) at $65^{\circ} \mathrm{C}$ under vigorous agitation.

Molecular cloning and amplification of plasmids were performed according to standard molecular biology methods.

\section{Construction of Pyrimidine Prototroph Strain}

Uracil prototroph strain was obtained by genomic integration of the pyrE2 gene at the HVO_0569 locus ${ }^{1}$ using the pop-in strategy (Allers et al., 2004) (Supplementary Figure S2). For homologous recombination, $500 \mathrm{bp}$ of the upstream and downstream regions spanning the HVO_0569 open reading frame were amplified by PCR using the following primers: us: oHv_025: 5'-GCATCGAGGGTACCTCGTCGTGCGAATCGCGGACG-3' and oHv_026: 5'-AGCGGAGGGGTCTCGACGGCGGATCC GCACGTCCGTCCGTCGGCGA-3'; ds: oHv_027 : 5'-TCGCC GACGGACGGACGTGCGGATCCGCCGTCGAGACCCCTCC GCT-3' / oHv_028: 5' -CTAGCCACTCTAGAGACCTCGCGCTC GACGGCGTGGC-3' (underlined nucleotides indicate the restriction enzyme sites used for cloning), and cloned into the integrative vector (pTA131) containing the pyrE2 gene as selection marker, as previously described (Allers et al., 2004). The resulting integrative plasmid was transformed in $\mathrm{H} 26$

\footnotetext{
${ }^{1}$ http://archaea.ucsc.edu/cgi-bin/hgGateway?db=haloVolc1
} 


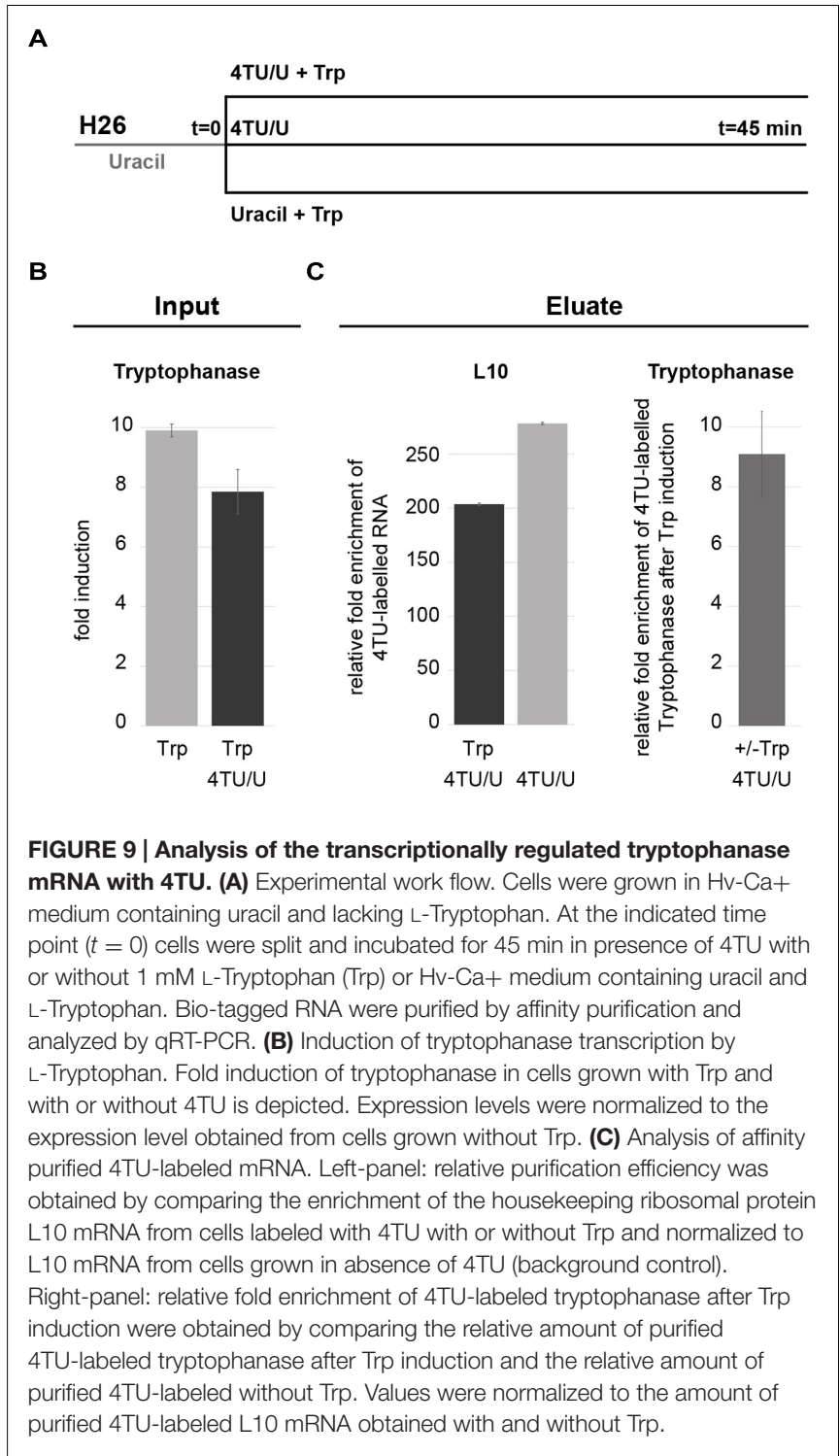

cells as previously described (Allers et al., 2004), and positive transformants were selected on $\mathrm{Hv}-\mathrm{Ca}^{+}$plates lacking uracil.

\section{4-TU Toxicity Analysis}

Semi-automated growth analysis was performed as previously described (Jantzer et al., 2011). In brief, exponentially growing cells $\left(\mathrm{Hv}-\mathrm{Ca}^{+}\right.$containing uracil) were diluted in $\mathrm{Hv}-\mathrm{Ca}^{+}$ media containing various relative amounts of $4 \mathrm{TU} /$ uracil (final concentration $400 \mu \mathrm{M})$ and aliquoted into a 96 -well plates. Growth $\left(\mathrm{OD}_{612 \mathrm{~nm}}\right)$ at $41.5^{\circ} \mathrm{C}\left( \pm 0.3^{\circ} \mathrm{C}\right)$ was monitored every $20 \mathrm{~min}$ for at least 3 days, using a TECAN Infinite F500 reader. Optical density values were corrected with the average background optical density measurement of abiotic medium. Biological replicates were performed in triplicate.

Growth analyses of $S$. acidocaldarius were performed manually as follows: exponentially growing cells (Brock medium supplemented with uracil) (Brock et al., 1972; Wagner et al., 2012) were diluted in pre-warmed Brock medium containing various relative amounts of $4 \mathrm{TU} /$ uracil (final concentration $180 \mu \mathrm{M}$ ) and incubated at $65^{\circ} \mathrm{C}$ with agitation. Optical density $\left(\mathrm{OD}_{600 \mathrm{~nm}}\right)$ was measured at regular time interval.

\section{Labeling with 4TU}

For $H$. volcanii, WT cells H26 (uracil auxotroph - $\Delta$ pyrE2) and "pop-in" mutants (uracil prototroph - pyrE2) were first grown in $\mathrm{Hv}-\mathrm{Ca}^{+}$medium in presence or absence of uracil $(400 \mu \mathrm{M})$, respectively (H26: +URA [400 $\mu \mathrm{M}$ ], “pop-in": -ura).

For 4TU pulse labeling experiments, exponentially growing cells were then transferred to fresh $\mathrm{Hv}-\mathrm{Ca}^{+}$media containing the indicated amount of 4TU [typically 75\% 4TU (300 $\mu \mathrm{M})$ and 25\% uracil $(100 \mu \mathrm{M})]$.

For pulse-chase experiments, cells were first grown in presence of $4 \mathrm{TU}$ and transferred to media $\mathrm{Hv}-\mathrm{Ca}^{+}$supplemented with uracil and lacking 4TU for the indicated time.

For S. acidocaldarius, MW001 cells were grown in Brock media supplemented with uracil (180 $\mu \mathrm{M})$ (Brock et al., 1972; Wagner et al., 2012). For pulse labeling experiments, exponentially growing cells were transferred to pre-warmed Brock media containing 75\% 4TU (135 $\mu \mathrm{M})$ and 25\% uracil (45 $\mu \mathrm{M})$.

For each sample time point, 2 ODs $\left(\mathrm{OD}_{600 \mathrm{~nm}}\right)$ were collected from cultures ranging between 0.4 and $0.8 \mathrm{OD}\left(\mathrm{OD}_{600 \mathrm{~nm}}\right)$, centrifuged and frozen or immediately processed further.

\section{Total RNA Extraction}

Total RNA was extracted using the hot-phenol extraction procedure as previously described (Schmitt et al., 1990).

\section{RNA Biotinylation}

For RNA biotinylation, typically 20-100 $\mu \mathrm{g}$ total RNA was labeled in the dark in presence of $50 \mu \mathrm{g}$ HPDP-biotin (Pierce) or $5 \mu \mathrm{g}$ MTSEA-biotin-XX (Biotium - 90066) in $10 \mathrm{mM}$ Tris$\mathrm{HCl} \mathrm{pH} \mathrm{7.4;} 1$ mM EDTA pH 8 for $2 \mathrm{~h}$ (HPDP-biotin) or $30 \mathrm{~min}$ (MTSEA-biotin-XX) (Duffy et al., 2015). Biotinylated RNAs were purified by the hot-phenol extraction procedure (Schmitt et al., 1990).

\section{Slot Blot and Northern Blot Analysis}

Total RNA (typically 5-10 $\mu \mathrm{g}$ ) was either separated by denaturing agarose gel electrophoresis in the absence of ethidium bromide or Syber-safe and transferred onto a positively charged nylon membrane (Ferreira-Cerca et al., 2005) or directly immobilized on nylon membrane using a vacuum slot-blotter (Millipore). RNAs were UV cross-linked twice with $0.5 \mathrm{~J} / \mathrm{cm} 2$.

\section{Detection of RNA}

For radioactive detection of bulk steady-state rRNA the following radiolabeled probes oHv194 $5^{\prime}-32$ P-CCTCGGCTG ATTGACAGTGCC-3', and Saci_022 $5^{\prime}-{ }^{32}$ P-CCTTTCGGTC GCCCCTACTC-3', antisense of the mature $H$. volcanii and S. acidocaldarius $23 \mathrm{~S} \mathrm{rRNA}$, respectively, were used. ${ }^{32} \mathrm{P}$ labeling of oligo probes, blot hybridization, and radioactive signal acquisition were performed as previously described (FerreiraCerca et al., 2005). 
For fluorescent detection of 4TU labeled RNA, membranes were blocked for $20 \mathrm{~min}$ in blocking solution (1X PBS $\mathrm{pH}$ 7.5, 1 mM EDTA $\mathrm{pH}$ 8) containing 10\% (w/v) SDS under mild agitation. Subsequently, the membranes were incubated at room temperature with IR-dye conjugated Streptavidin (1:10,000 dilution in blocking solution containing 10\% SDS - IRDye 800CW Streptavidin, Pierce) for $20 \mathrm{~min}$ in the dark under mild agitation. Membranes were then washed with blocking solution containing decreasing amounts of SDS, twice each with 10, 1, and $0.1 \%$ SDS, for 10 min each. Labeled RNAs were visualized using a LI-COR Odyssey imaging platform.

\section{Relative Quantification}

Fluorescence and radioactive signals were quantified with ImageJ. 4TU-labeled 23S rRNA signal intensity (fluorescence signal) were normalized to the bulk 23S rRNA signal (radioactive signal).

\section{Affinity Purification of Bio-Tagged-RNA}

Exponentially growing wild type $H$. volcanii cells (H26) were diluted into pre-warmed enhanced casamino acids supplemented either with uracil and $1 \mathrm{mM}$ tryptophan or supplemented with 4TU/uracil with or without $1 \mathrm{mM}$ tryptophan. After $45 \mathrm{~min}$ of incubation $4 \mathrm{OD}_{600 \mathrm{~nm}}$ of cells were harvested. Total RNA was extracted as described above. Residual genomic DNA was digested in presence of RQ1 DNAse (Promega) and RNasin (NEB) as recommended by the manufacturer. The RNA was purified by hot-phenol extraction and biotinylated as described above. Purified RNA (25 $\mu \mathrm{g})$ was resuspended in buffer B $(20 \mathrm{mM}$ Tris- $\mathrm{HCl}$ pH 7.6, $150 \mathrm{mM} \mathrm{NaCl}, 5 \mathrm{mM} \mathrm{MgCl} 2,1 \mathrm{mM}$ DTT) (Nielsen et al., 2011) and incubated for $2 \mathrm{~h}$ on a rotating wheel at $4^{\circ} \mathrm{C}$ with high-capacity Streptavidin agarose beads (Pierce) in a total volume of $800 \mu \mathrm{l}$ buffer B. Immobilized RNA was washed three times for $10 \mathrm{~min}$ on a rotating wheel at $4^{\circ} \mathrm{C}$ with $800 \mu \mathrm{l}$ buffer B. Competitive RNA elution was performed twice in batches using $500 \mu \mathrm{l}$ buffer B supplemented with $2.5 \mathrm{mM}$ Ddesthiobiotin (IBA) at 4 and $23^{\circ} \mathrm{C}$, respectively. RNA was purified using hot-phenol extraction as described above.

\section{Quantitative RT-PCR Analysis}

Reverse transcription (SuperScript III - Invitrogen) was performed according to the manufacturer's instructions in presence of $0.2 \mu \mathrm{g}$ random primers (Agilent). Complementary DNA synthesis was performed with a pre-incubation at $25^{\circ} \mathrm{C}$ followed by $60 \mathrm{~min}$ at $42^{\circ} \mathrm{C}$.

Quantitative PCR was performed with previously published primers, complementary to the tryptophanase ORF Trpase_R2 5'-ACACCGGTTCGAGCCGCGACG-3', Trpase_F2_RT 5'-TT CGCGTTCCCCGGCACCGAC-3' (202 bp) and the ribosomal protein L10 ORF RibL10-H.v. 5'-CCGGTCGCCT GCTTGTTCTCGCG-3', RibL10-H. v. 5'-CCGAGGACTACC CCGTCCAGATTAGCCTG- 3' (187 bp) (Large et al., 2007).

Each PCR reaction was performed in a $20 \mu \mathrm{l}$ reaction containing $2 \mu \mathrm{l}$ 10X PCR-Buffer (Qiagen), $0.8 \mu 125 \mathrm{mM} \mathrm{MgCl} 2$, $0.16 \mu l 25$ mM dNTP-mix, $0.08 \mu \mathrm{l}$ ( $5 \mathrm{U} / \mu \mathrm{l}$ ) HotStar Taq (Qiagen), $0.25 \mu \mathrm{l}$ SYBR green I dye stock (Roche, diluted in DMSO 1:400000), 4 pmol of each primer and $4 \mu$ l of template cDNA.
PCR reactions and SYBR green I dye fluorescence acquisition were performed with a Rotor-Gene 3000 system (Corbett Research/Qiagen). Relative quantification analysis was performed using the comparative analysis software module (Rotor-gene 6 - Corbett Research/Qiagen). Relative levels were calculated according to the $2^{-\Delta \Delta C_{T}}$ method (Livak and Schmittgen, 2001) and using the ribosomal protein L10 mRNA level as reference (Large et al., 2007). Serial dilutions of the samples were run in triplicate to ensure accuracy of the data.

\section{AUTHOR CONTRIBUTIONS}

$\mathrm{RK}, \mathrm{CK}$, and SF-C designed and performed all the experiments and interpreted the results. SF-C supervised the study and wrote the paper. All authors critically commented on the manuscript.

\section{FUNDING}

This work was supported by the department of Biochemistry III "House of the Ribosome" and by the DFG collaborative research center SFB960 "Ribosome formation: principles of RNP biogenesis and control of their function". This work was supported by the German Research Foundation (DFG) within the funding program Open Access Publishing.

\section{ACKNOWLEDGMENTS}

The authors would like to acknowledge Prof. Dr. Anita Marchfelder (University of Ulm), Dr. Thorsten Allers (University of Nottingham), Prof. Dr. Sonja-Verena Albers (University of Freiburg), Prof. Dr. Gunter Meister, Dr. Jan Medenbach, Daniela Strauß and all members of the Biochemistry department III "House of the Ribosome" (University of Regensburg) for sharing strains, reagents, protocols, instrumentations, and scientific discussions.

\section{SUPPLEMENTARY MATERIAL}

The Supplementary Material for this article can be found online at: http://journal.frontiersin.org/article/10.3389/fmicb. 2017.00286/full\#supplementary-material

FIGURE S1 | Detection of 4TU with HPDP-biotin and MTSEA-biotin-XX. $H$. volcanii $(\mathrm{H} 26)$ cells were grown for several generations either in medium containing a mixture of 4-thiouracil and uracil (4TU/U - 3:1) or in medium solely containing uracil (U). Nucleic acids were analyzed as described in the Section "Materials and Methods."

FIGURE S2 | Generation of uracil prototroph strain. The upstream and downstream region of the HVO_0569 open reading frame were amplified by PCR and cloned into the integrative vector pTA131 and transformed in H26 cells. Recombination events leading to genomic integration of the pyrE2 gene were selected on $\mathrm{Hv}-\mathrm{Ca}^{+}$plates lacking uracil. 


\section{REFERENCES}

Allers, T., Ngo, H.-P., Mevarech, M., and Lloyd, R. G. (2004). Development of additional selectable markers for the Halophilic Archaeon Haloferax volcanii based on the leuB and trpA genes. Appl. Environ. Microbiol. 70, 943-953. doi: 10.1128/AEM.70.2.943-953.2004

Ascano, M., Hafner, M., Cekan, P., Gerstberger, S., and Tuschl, T. (2012). Identification of RNA-protein interaction networks using PAR-CLIP. Wiley Interdiscip. Rev. RNA 3, 159-177. doi: 10.1002/wrna.1103

Atomi, H., Imanaka, T., and Fukui, T. (2012). Overview of the genetic tools in the Archaea. Front. Microbiol. 3:337. doi: 10.3389/fmicb.2012.00337

Barabasi, A.-L., and Oltvai, Z. N. (2004). Network biology: understanding the cell's functional organization. Nat. Rev. Genet. 5, 101-113. doi: 10.1038/nrg1272

Barrass, J. D., Reid, J. E. A., Huang, Y., Hector, R. D., Sanguinetti, G., Beggs, J. D., et al. (2015). Transcriptome-wide RNA processing kinetics revealed using extremely short 4tU labeling. Genome Biol. 16, 282. doi: 10.1186/s13059-0150848-1

Blais, A., and Dynlacht, B. D. (2005). Constructing transcriptional regulatory networks. Genes Dev. 19, 1499-1511. doi: 10.1101/gad.1325605

Bork, P., Dandekar, T., Diaz-Lazcoz, Y., Eisenhaber, F., Huynen, M., and Yuan, Y. (1998). Predicting function: from genes to genomes and back1. J. Mol. Biol. 283, 707-725. doi: 10.1006/jmbi.1998.2144

Brock, T. D., Brock, K. M., Belly, R. T., and Weiss, R. L. (1972). Sulfolobus: a new genus of sulfur-oxidizing bacteria living at low $\mathrm{pH}$ and high temperature. Arch. Mikrobiol. 84, 54-68. doi: 10.1007/BF00408082

Burger, K., Mühl, B., Kellner, M., Rohrmoser, M., Gruber-Eber, A., Windhager, L., et al. (2013). 4-thiouridine inhibits rRNA synthesis and causes a nucleolar stress response. RNA Biol. 10, 1623-1630. doi: 10.4161/rna.26214

Castello, A., Fischer, B., Eichelbaum, K., Horos, R., Beckmann, B. M., Strein, C., et al. (2012). Insights into RNA biology from an Atlas of Mammalian mRNA-binding proteins. Cell 149, 1393-1406. doi: 10.1016/j.cell.2012. 04.031

Chavarria, N. E., Hwang, S., Cao, S., Fu, X., Holman, M., Elbanna, D., et al. (2014). Archaeal Tuc1/Ncs6 homolog required for wobble uridine tRNA thiolation is associated with ubiquitin-proteasome, translation, and RNA processing system homologs. PLOS ONE 9:e99104. doi: 10.1371/journal.pone. 0099104

Cleary, M. D., Meiering, C. D., Jan, E., Guymon, R., and Boothroyd, J. C. (2005). Biosynthetic labeling of RNA with uracil phosphoribosyltransferase allows cellspecific microarray analysis of mRNA synthesis and decay. Nat. Biotechnol. 23, 232-237. doi: 10.1038/nbt1061

Dölken, L., Ruzsics, Z., Rädle, B., Friedel, C. C., Zimmer, R., Mages, J., et al. (2008). High-resolution gene expression profiling for simultaneous kinetic parameter analysis of RNA synthesis and decay. RNA 14, 1959-1972. doi: 10.1261/rna. 1136108

Duffy, E. E., Rutenberg-Schoenberg, M., Stark, C. D., Kitchen, R. R., Gerstein, M. B., and Simon, M. D. (2015). Tracking distinct RNA populations using efficient and reversible covalent chemistry. Mol. Cell 59, 858-866. doi: 10.1016/j.molcel.2015. 07.023

Edmonds, C. G., Crain, P. F., Gupta, R., Hashizume, T., Hocart, C. H., Kowalak, J. A., et al. (1991). Posttranscriptional modification of tRNA in thermophilic archaea (Archaebacteria). J. Bacteriol. 173, 3138-3148.

Favre, A., Bezerra, R., Hajnsdorf, E., Dubreuil, Y. L., and Expert-Besancon, A. (1986). Substitution of uridine in vivo by the intrinsic photoactivable probe 4thiouridine in Escherichia coli RNA. Eur. J. Biochem. 160, 441-449. doi: 10.1111/ j.1432-1033.1986.tb10060.x

Ferreira-Cerca, S., Pöll, G., Gleizes, P.-E., Tschochner, H., and Milkereit, P. (2005). Roles of eukaryotic ribosomal proteins in maturation and transport of Pre-18S rRNA and ribosome function. Mol. Cell 20, 263-275. doi: 10.1016/j.molcel.2005. 09.005

Fraser, A. G., and Marcotte, E. M. (2004). A probabilistic view of gene function. Nat. Genet. 36, 559-564. doi: 10.1038/ng1370

Friedel, C. C., and Dolken, L. (2009). Metabolic tagging and purification of nascent RNA: implications for transcriptomics. Mol. Biosyst. 5, 1271-1278. doi: 10.1039/ B911233B

Gardner, T. S., di Bernardo, D., Lorenz, D., and Collins, J. J. (2003). Inferring genetic networks and identifying compound mode of action via expression profiling. Science 301, 102-105. doi: 10.1126/science.1081900
Hafner, M., Landthaler, M., Burger, L., Khorshid, M., Hausser, J., Berninger, P., et al. (2010). Transcriptome-wide identification of RNA-binding protein and MicroRNA target sites by PAR-CLIP. Cell 141, 129-141. doi: 10.1016/j.cell.2010. 03.009

Hatzenpichler, R., Connon, S. A., Goudeau, D., Malmstrom, R. R., Woyke, T., and Orphan, V. J. (2016). Visualizing in situ translational activity for identifying and sorting slow-growing archaeal-bacterial consortia. Proc. Natl. Acad. Sci. U.S.A. 113, E4069-E4078. doi: 10.1073/pnas.1603757113

Hatzenpichler, R., Scheller, S., Tavormina, P. L., Babin, B. M., Tirrell, D. A., and Orphan, V. J. (2014). In situ visualization of newly synthesized proteins in environmental microbes using amino acid tagging and click chemistry. Environ. Microbiol. 16, 2568-2590. doi: 10.1111/1462-2920.12436

Hulscher, R. M., Bohon, J., Rappé, M. C., Gupta, S., D’Mello, R., Sullivan, M., et al. (2016). Probing the structure of ribosome assembly intermediates in vivo using DMS and hydroxyl radical footprinting. Adv. RNA Struct. Determ. 103, 49-56. doi: 10.1016/j.ymeth.2016.03.012

Ihmels, J., Bergmann, S., and Barkai, N. (2004). Defining transcription modules using large-scale gene expression data. Bioinformatics 20, 1993-2003. doi: 10. 1093/bioinformatics/bth166

Jantzer, K., Zerulla, K., and Soppa, J. (2011). Phenotyping in the archaea: optimization of growth parameters and analysis of mutants of Haloferax volcanii. FEMS Microbiol. Lett. 322, 123-130. doi: 10.1111/j.1574-6968.2011. 02341.x

Kanehisa, M., Sato, Y., Kawashima, M., Furumichi, M., and Tanabe, M. (2016). KEGG as a reference resource for gene and protein annotation. Nucleic Acids Res. 44, D457-D462. doi: 10.1093/nar/gkv1070

Large, A., Stamme, C., Lange, C., Duan, Z., Allers, T., Soppa, J., et al. (2007). Characterization of a tightly controlled promoter of the halophilic archaeon Haloferax volcanii and its use in the analysis of the essential cct1 gene. Mol. Microbiol. 66, 1092-1106. doi: 10.1111/j.1365-2958.2007. 05980.x

Liu, Y., Zhu, X., Nakamura, A., Orlando, R., Söll, D., and Whitman, W. B. (2012). Biosynthesis of 4-thiouridine in tRNA in the methanogenic archaeon Methanococcus maripaludis. J. Biol. Chem. 287, 36683-36692. doi: 10.1074/jbc. M112.405688

Livak, K. J., and Schmittgen, T. D. (2001). Analysis of relative gene expression data using real-time quantitative PCR and the $2^{-\Delta \Delta C_{\mathrm{T}}}$ method. Methods 25, 402-408. doi: 10.1006/meth.2001.1262

Machnicka, M. A., Olchowik, A., Grosjean, H., and Bujnicki, J. M. (2014). Distribution and frequencies of post-transcriptional modifications in tRNAs. RNA Biol. 11, 1619-1629. doi: 10.4161/15476286.2014.992273

Miller, M. R., Robinson, K. J., Cleary, M. D., and Doe, C. Q. (2009). TU-tagging: cell type-specific RNA isolation from intact complex tissues. Nat. Meth. 6, 439-441. doi: 10.1038/nmeth.1329

Miranda, H. V., Nembhard, N., Su, D., Hepowit, N., Krause, D. J., Pritz, J. R., et al. (2011). E1- and ubiquitin-like proteins provide a direct link between protein conjugation and sulfur transfer in archaea. Proc. Natl. Acad. Sci. U.S.A. 108, 4417-4422. doi: 10.1073/pnas.1018151108

Niehrs, C., and Pollet, N. (1999). Synexpression groups in eukaryotes. Nature 402, 483-487. doi: 10.1038/990025

Nielsen, K. H., Behrens, M. A., He, Y., Oliveira, C. L. P., Sottrup Jensen, L., Hoffmann, S. V., et al. (2011). Synergistic activation of eIF4A by eIF4B and eIF4G. Nucleic Acids Res. 39, 2678-2689. doi: 10.1093/nar/ gkq1206

Phillips, G., and de Crécy-Lagard, V. (2011). Biosynthesis and function of tRNA modifications in Archaea. Curr. Opin. Microbiol. 14, 335-341. doi: 10.1016/j. mib.2011.03.001

Rogers, K. C., Crescenzo, A. T., and Söll, D. (1995). Aminoacylation of transfer RNAs with 2-thiouridine derivatives in the wobble position of the anticodon. Biochimie 77, 66-74. doi: 10.1016/0300-9084(96)88106-5

Schlitt, T., Palin, K., Rung, J., Dietmann, S., Lappe, M., Ukkonen, E., et al. (2003). From gene networks to gene function. Genome Res. 13, 2568-2576. doi: 10. $1101 /$ gr.1111403

Schmitt, M. E., Brown, T. A., and Trumpower, B. L. (1990). A rapid and simple method for preparation of RNA from Saccharomyces cerevisiae. Nucleic Acids Res. 18, 3091-3092.

Shigi, N. (2014). Biosynthesis and functions of sulfur modifications in tRNA. Front. Genet. 5:67. doi: 10.3389/fgene.2014.00067 
Swiatkowska, A., Wlotzka, W., Tuck, A., Barrass, J. D., Beggs, J. D., and Tollervey, D. (2012). Kinetic analysis of pre-ribosome structure in vivo. RNA 18, 2187-2200. doi: 10.1261/rna.034751.112

Tallafuss, A., Washbourne, P., and Postlethwait, J. (2014). Temporally and spatially restricted gene expression profiling. Curr. Genomics 15, 278-292. doi: 10.2174/ 1389202915666140602230106

Tomikawa, C., Ohira, T., Inoue, Y., Kawamura, T., Yamagishi, A., Suzuki, T., et al. (2013). Distinct tRNA modifications in the thermo-acidophilic archaeon, Thermoplasma acidophilum. FEBS Lett. 587, 3575-3580. doi: 10.1016/j.febslet. 2013.09.021

Wagner, M., van Wolferen, M., Wagner, A., Lassak, K., Meyer, B. H., Reimann, J., et al. (2012). Versatile genetic tool box for the crenarchaeote Sulfolobus acidocaldarius. Front. Microbiol. 3:214. doi: 10.3389/fmicb.2012.00214

Wang, Z., Gerstein, M., and Snyder, M. (2009). RNA-Seq: a revolutionary tool for transcriptomics. Nat. Rev. Genet. 10, 57-63. doi: 10.1038/nrg2484

Wu, L. F., Hughes, T. R., Davierwala, A. P., Robinson, M. D., Stoughton, R., and Altschuler, S. J. (2002). Large-scale prediction of Saccharomyces cerevisiae gene function using overlapping transcriptional clusters. Nat. Genet. 31, 255-265. doi: $10.1038 /$ ng906

Zeiner, G. M., Cleary, M. D., Fouts, A. E., Meiring, C. D., Mocarski, E. S., and Boothroyd, J. C. (2008). "RNA analysis by biosynthetic tagging using 4-thiouracil and uracil phosphoribosyltransferase," in Post-Transcriptional Gene Regulation, ed. J. Wilusz (Totowa, NJ: Humana Press), 135-146.

Conflict of Interest Statement: The authors declare that the research was conducted in the absence of any commercial or financial relationships that could be construed as a potential conflict of interest.

Copyright (c) 2017 Knüppel, Kuttenberger and Ferreira-Cerca. This is an open-access article distributed under the terms of the Creative Commons Attribution License (CC BY). The use, distribution or reproduction in other forums is permitted, provided the original author(s) or licensor are credited and that the original publication in this journal is cited, in accordance with accepted academic practice. No use, distribution or reproduction is permitted which does not comply with these terms. 